\title{
Desfechos clínicos de pacientes neurológicos com e sem ventilação mecânica prolongada
}

\section{Clinical outcomes of neurological patients with and without prolonged mechanical ventilation}

\section{Lucas Lima Ferreira' ${ }^{1}$ Anna Carolina Macedo Sousa ${ }^{2}$ (1) Lilian Cristina Ascencio Sanchez ${ }^{3}$ (1)}

${ }^{1}$ Autor para correspondência. União das Faculdades dos Grandes Lagos (São José do Rio Preto), São Paulo, Brasill. lucas_lim21@hotmail.com ${ }^{2-3}$ Faculdade de Medicina de São José do Rio Preto (São José do Rio Preto). São Paulo, Brasil. carolina.macedo.sousa@hotmail.com, lilian.sanchez_@hotmail.com

RESUMO | INTRODUÇÃO: Pacientes com doenças neurológicas sob ventilação mecânica (VM) apresentam maior risco de VM prolongada (VMP) devido ao rebaixamento do nível de consciência, padrões respiratórios anormais e incapacidade de proteção de vias aéreas causados pela lesão neurológica. OBJETIVo: Comparar desfechos clínicos de pacientes neurológicos com ou sem VMP. MATERIAIS E MÉTODOS: Tratou-se de um estudo observacional documental retrospectivo, realizado na UTI neurológica de um hospital escola. Foram coletados: idade, sexo, patologia que motivou a internação, presença de comorbidades, valor do simplified acute physiology score (SAPS III), incidência de VMP, tempo de permanência em VM, tempo de permanência na UTI e desfecho alta ou óbito na unidade. Os pacientes foram divididos em dois grupos, de acordo com a duração da VM: o grupo VMP e o grupo sem VMP. RESULTADOS: Foram incluídos 212 pacientes. 0 diagnóstico mais prevalente no grupo VMP foi a ressecção de tumor cerebral $(27,5 \%)$ e no grupo sem VMP foi o traumatismo cranioencefálico (18\%). Verificouse que $10 \%$ desses evoluíram para VMP, houve prevalência do sexo masculino em ambos os grupos. O grupo VMP apresentou escore SAPS III significativamente maior $(p=0,003)$ que o grupo sem VMP. $O$ grupo VMP permaneceu tempo, em dias, significativamente maior $(p<0,0001)$ em VM e em internação na UTI que o grupo sem VMP. Não houve diferença significativa $(p=1,00)$ no desfecho alta ou óbito da UTI entre os grupos com e sem VMP. CONCLUSÃO: $O$ grupo de pacientes sob VMP esteve mais tempo em suporte ventilatório invasivo e permaneceu mais tempo internado na UTI que o grupo sem VMP. Não houve diferenças estatísticas no desfecho mortalidade na UTI.

PALAVRAS-CHAVE: Respiração Artificial. Unidades de Terapia Intensiva. Desmame do Respirador.
ABSTRACT | INTRODUCTION: Patients with neurological diseases on mechanical ventilation (MV) are at increased risk of prolonged MV (PMV) due to lowered level of consciousness, abnormal breathing patterns, and inability to protect the airways caused by neurological damage. OBJECTIVE: To compare clinical outcomes of neurological patients with or without PVM. MATERIALS AND METHODS: This was a retrospective observational documentary study carried out in the neurological ICU of a teaching hospital. The following were collected: age, gender, the pathology that led to hospitalization, presence of comorbidities, simplified acute physiology score (SAPS III), the incidence of PMV, length of stay on MV, length of stay in the ICU, and outcome discharge or death in the unit. Patients were divided into two groups according to the duration of MV, the PMV group and the non-PMV group. RESULTS: A total of 212 patients were included, the most prevalent diagnosis in the PMV group was brain tumor resection (27.5\%), and in the group without PMV, it was traumatic brain injury (18\%). It was found that $10 \%$ of these evolved to PMV. There was a prevalence of males in both groups. The PMV group had a significantly higher SAPS III score $(p=0.003)$ than the group without PMV. The PMV group remained significantly longer $(p<0.0001)$ in MV and ICU stay in days than the group without PMV. There was no significant difference $(p=1.00)$ in the ICU outcome of discharge or death between the groups with and without PMV. CONCLUSION: The group of patients under PMV spent more time on invasive ventilatory support and remained in the ICU longer than those without PMV. There were no statistical differences in the ICU mortality outcome.

KEYWORDS: Artificial Respiration. Intensive Care Units. Respirator. Weaning. 


\section{Introdução}

De acordo com a literatura, 40\% dos pacientes internados em unidade de terapia intensiva (UTI) são submetidos à ventilação mecânica (VM) invasiva ${ }^{1}$, destes, $20 \%$ são pacientes com distúrbios neurológicos agudos, como disfunção do sistema nervoso central, traumas ou coma, sendo estas as principais razões para necessidade do suporte ventilatório invasivo.?

A maioria dos pacientes sob VM necessita de curtos períodos de suporte ventilatório, mas uma minoria demanda VM prolongada (VMP), que é definida como um período maior que 24 horas ou mais de 96 horas consecutivas ${ }^{3}$, ou ainda, mais de sete dias. ${ }^{4}$ Loss et al. ${ }^{5}$ e Chelluri et al. ${ }^{-}$utilizaram a definição de VMP como maior ou igual a seis horas/dia por 21 dias. Dessa forma, verifica-se a heterogeneidade de definições de VMP na literatura, sendo assim, para o presente estudo, utilizou-se a definição de VMP como superior a sete dias em pacientes neurocirúrgicos. A VMP está diretamente associada com aumento da mortalidade precoce e, em longo prazo, pior qualidade de vida, maiores demandas e gastos com assistência à saúde. ${ }^{\text {? }}$

A incongruência nas definições e critérios de VMP geram uma variação na literatura acerca dos dados epidemiológicos e dos desfechos clínicos. $\stackrel{-}{ }$ Algumas evidências literárias apontam incidência de VMP entre 3-14\%. 910 Existe uma lacuna importante de dados epidemiológicos robustos de pacientes em VMP internados em UTIs brasileiras, principalmente devido à ausência de unidades especializadas em desmame ventilatório. ${ }^{4}$

Ademais, pacientes com doenças neurológicas, submetidos à VM, apresentam maior risco de pneumonias associadas à ventilação (PAV), traqueostomias, tempo prolongado em suporte ventilatório e pior desfecho na UTI. ${ }^{11,12}$ Esses fatores de risco nos pacientes neurológicos ocorrem devido à lesão anatômica do sistema nervoso central, que pode provocar alterações na complacência cerebral, resultando em rebaixamento do nível de consciência, padrões respiratórios anormais e incapacidade de proteção de vias aéreas..$^{11}$

Nesse contexto, identificar a prevalência, bem como os resultados de pacientes sob VMP em UTI neurológica se torna preponderante para delinear estratégias de prevenção e enfrentamento desta condição.
Assim, este estudo objetivou comparar desfechos clínicos de pacientes neurológicos com ou sem VMP. $E$, como objetivo específico, avaliar a incidência de VMP. A hipótese do presente estudo é que pacientes neurológicos que permanecem em VMP apresentarão piores desfechos clínicos, como maior tempo de internação e maior mortalidade na UTI.

\section{Materiais e métodos}

Foi realizado um estudo observacional documental retrospectivo, realizado na UTI Neurológica do $7^{\circ}$ andar do Hospital de Base da Fundação Faculdade Regional de Medicina (FUNFARME), na cidade de São José de Rio Preto - SP, Brasil, com dados referentes ao período de junho de 2014 a abril de 2020.

Os dados foram coletados do Livro de Desmame Ventilatório do Serviço de Fisioterapia e dos prontuários clínicos eletrônicos via sistema MVPEP® dos pacientes da UTI neurológica do referido hospital. A coleta de dados foi realizada nos meses de maio e junho de 2020. O desfecho clínico primário foi a mortalidade na unidade e o desfecho secundário foi o tempo de internação na UTI.

Foram incluídos no estudo dados de pacientes com idade maior ou igual a 18 anos que estiveram sob VM a mais de 24 horas na UTI. Prontuários de pacientes com doença neuromuscular conhecida, além daqueles com dados incompletos ou imprecisos sobre a duração da ventilação antes da admissão à UTI, ou que tivessem restrição ou suspensão de medidas terapêuticas durante a permanência na UTI foram excluídos do estudo.

Para a proposta deste estudo, a VMP foi definida como suporte ventilatório invasivo por mais de sete dias, baseada em evidências da literatura que apontam que para pacientes cirúrgicos, tempo superior a 48 horas já pode ser definido como VMP. 13 Os pacientes foram divididos em dois grupos, com base na duração da VM, o grupo com ventilação prolongada, ou seja, o grupo que permaneceu tempo igual ou superior a sete dias em VM e o grupo sem ventilação prolongada, ou seja, o grupo que permaneceu menos de sete dias em VM. 
Foram coletados dados como idade, sexo, patologia que o levou a internação, presença de comorbidades como hipertensão arterial, diabetes mellitus, etilismo, tabagismo, entre outras, valor do Simplified acute physiology score (SAPS III), tempo de permanência em dias em VM, tempo de permanência em dias na UTI, e desfechos na unidade como alta ou óbito, em formulário próprio estruturado pelos pesquisadores.

O escore SAPS III é uma ferramenta formada por 20 componentes de avaliação quando o paciente é admitido na UTI. Entre os componentes do escore, estão informações demográficas, motivo da admissão, variáveis fisiológicas e as condições de saúde anteriores à internação hospitalar. Para cada componente atribui-se um valor, conforme a gravidade do sistema fisiológico. O escore total varia de 16 a 217 pontos. 14,15

O trabalho seguiu os princípios éticos estabelecidos na Resolução n. ${ }^{\circ}$ 466/2012 do Conselho Nacional em Saúde e foi submetido ao Comitê de Ética em Pesquisa da União das Faculdades dos Grandes Lagos - UNILAGO (CAAE: 31914620.8.0000.5489) e aprovado sob parecer n. ${ }^{\circ}$ 4.062.514. Foi solicitada e autorizada dispensa do termo de consentimento livre e esclarecido junto ao comitê de ética, dado o desenho metodológico documental do estudo.

A análise estatística foi realizada de forma descritiva, em que as variáveis contínuas foram apresentadas em forma de média \pm desvio-padrão ou mediana (variação interquartil) e as variáveis categóricas foram descritas em porcentagens e frequências. Foi analisada normalidade dos dados por meio do teste de Kolmogorov-Smirnov. Foi realizada análise estatística inferencial por meio do teste $t$ de Student não pareado ou teste de Mann-Whitney para comparação das variáveis contínuas e teste exato de Fisher para comparação das variáveis categóricas. Para isto foi utilizado o programa Statistical Package for Social Sciences (SPSS) e foram considerados estatisticamente significativos valores de $p \leq 0,05$.

\section{Resultados}

Foram encontrados 238 registros de desmame ventilatório no livro de desmame do serviço de fisioterapia, dos quais foram incluídos 212 (Figura 1).

Figura 1. Fluxograma de seleção da amostra.

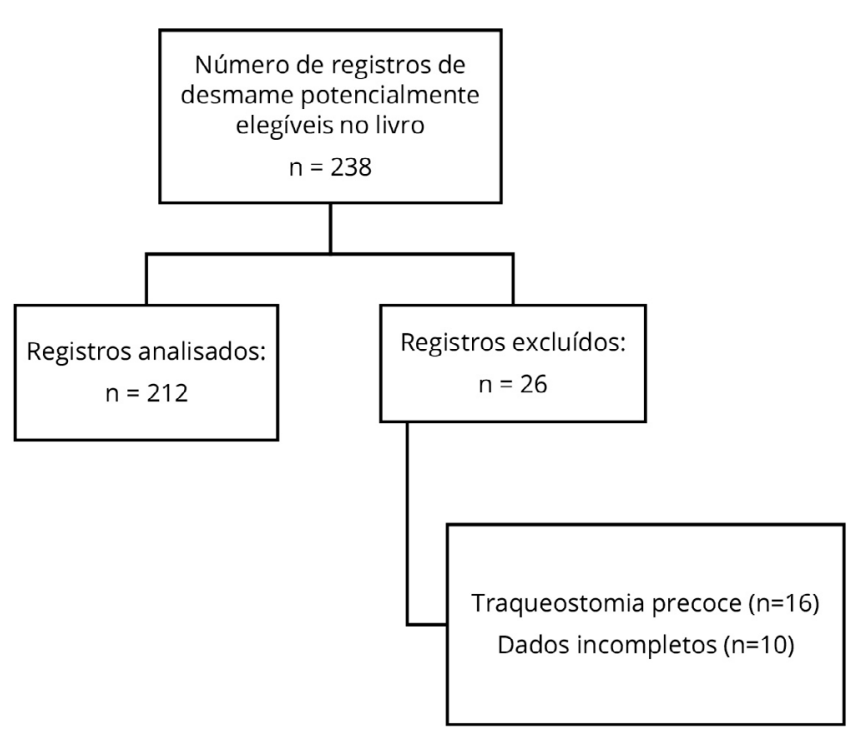


Verificou-se incidência de VMP de 10\% ( $n=21$ ), e prevalência do sexo masculino em ambos os grupos com diferença estatisticamente significativa entre os grupos sem VMP e com VMP $(p=0,03)$ entre o sexo masculino e o feminino. Além disso, o grupo com VMP apresentou escore SAPS III significativamente maior $(p=0,003)$ que o grupo sem VMP (Tabela 1). Entre os diagnósticos de admissão mais prevalentes, verificou-se o traumatismo cranioencefálico $(18 \% / n=21)$ no grupo sem VMP e a ressecção de tumor cerebral $(27,5 \% / n=11)$ no grupo com VMP. Também verificaram-se outros diagnósticos nos grupos sem $(21 \% / n=25)$ e com VMP $(15 \% / n=6)$, os quais incluíram artrodeses de coluna vertebral, choques sépticos de origem meningocócica, hidrocefalias, etc. (Tabela 1).

Tabela 1. Características sociodemográficas e clínicas dos pacientes

\begin{tabular}{|c|c|c|c|}
\hline Variável & Grupo sem VMP & Grupo com VMP & p-valor \\
\hline Amostra & $\mathrm{n}=190(90 \%)$ & $n=22(10 \%)$ & - \\
\hline \multirow[t]{2}{*}{ Sexo-M/F } & $M=112(59 \%)$ & $M=18(82 \%)$ & $0,03 t$ \\
\hline & $\mathrm{F}=78(41 \%)$ & $F=04(18 \%)$ & \\
\hline Idade (anos) & $51,1 \pm 15,7$ & $48,9 \pm 16,3$ & $0,53^{*}$ \\
\hline SAPS III & $54,3 \pm 17,4$ & $68,8 \pm 14,6$ & $0,003^{*}$ \\
\hline \multicolumn{4}{|l|}{ Diagnóstico } \\
\hline AVC & $n=15(13 \%)$ & $n=8(20 \%)$ & \\
\hline HSA aneurismática & $n=17(14,5 \%)$ & $n=7(17,5 \%)$ & \\
\hline Ressecção de tumor cerebral & $n=16(13,5 \%)$ & $n=11(27,5 \%)$ & \\
\hline Politrauma & $n=15(13 \%)$ & $n=3(7,5 \%)$ & \\
\hline TCE & $n=21(18 \%)$ & $n=5(12,5 \%)$ & \\
\hline Drenagem de hematoma cerebral & $\mathrm{n}=9(8 \%)$ & - & \\
\hline Outros & $n=25(21 \%)$ & $n=6(15 \%)$ & \\
\hline
\end{tabular}

M: masculino; F: feminino. SAPS: Simplified acute physiology score. VMP: ventilação mecânica prolongada. AVC: acidente vascular cerebral; HSA: hemorragia subaracnóidea; TCE: traumatismo cranioencefálico. tteste exato de Fisher; *teste t não pareado.

Entre as comorbidades mais prevalentes verificou-se a hipertensão arterial sistêmica em ambos os grupos sem (36\%) e com VMP (35\%), seguidas de etilismo e tabagismo (Tabela 2).

Tabela 2. Comorbidades mais prevalentes nos pacientes dos grupos com e sem VMP

\begin{tabular}{lcccc}
\hline \multicolumn{1}{c}{ Comorbidade } & \multicolumn{2}{c}{ Grupo sem VMP } & \multicolumn{2}{c}{ Grupo com VMP } \\
& $\mathbf{n}$ & $\mathbf{\%}$ & $\mathbf{n}$ & $\mathbf{\%}$ \\
\hline Hipertensão arterial sistêmica & 43 & $\mathbf{3 6}$ & 14 & $\mathbf{3 5}$ \\
Diabetes mellitus & 13 & 11 & 4 & 5 \\
Obesidade & 3 & 2,5 & 2 & - \\
Dislipidemias & 6 & 5 & 2 & 5 \\
Doença renal crônica & 3 & 2,5 & 10 & 25 \\
Etilismo & 25 & 21 & 10 & 30 \\
Tabagismo & 25 & 21 & 12 & 5 \\
\hline
\end{tabular}

VMP: ventilação mecânica prolongada. 
Verificou-se que o grupo com VMP permaneceu mediana de tempo de VM de sete dias enquanto o grupo sem VMP, mediana de apenas um dia. Em relação a variável de desfecho tempo de internação na UTI, o grupo com VMP permaneceu tempo significativamente maior $(p<0,0001)$ que o grupo sem VMP (Tabela 3$)$.

Tabela 3. Comparação das variáveis de desfecho dos grupos com e sem VMP

\begin{tabular}{lccc}
\hline Variável & Grupo sem VMP & Grupo com VMP & p-valor \\
\hline Internação na UTI (dias) & $8[2-40]$ & $17,5[8-49]$ & $<0,0001^{*}$ \\
Desmame da VM (Sucesso/Falha) & $\mathrm{S}=150(79 \%)$ & $\mathrm{S}=14(64 \%)$ & $0,11 \dagger$ \\
& $\mathrm{F}=40(21 \%)$ & $\mathrm{F}=8(36 \%)$ & \\
Desfecho na UTI (Alta/Óbito) & $\mathrm{A}=162(85 \%)$ & $\mathrm{A}=19(86 \%)$ & $1,00 \dagger$ \\
& $\mathrm{O}=28(15 \%)$ & $\mathrm{O}=3(14 \%)$ & \\
\hline
\end{tabular}

VM: ventilação mecânica; VMP: ventilação mecânica prolongada. S: sucesso; F: falha; A: alta; O: óbito. *teste de MannWhitney. tteste exato de Fisher.

\section{Discussão}

No presente estudo a incidência de VMP nos pacientes com doenças neurológicas foi baixa e na comparação das variáveis de desfecho, não foi observada diferença significativa na mortalidade e alta entre os grupos, porém, foi identificado que o grupo VMP apresentou um tempo de internação na UTI superior que o grupo sem VMP.

A baixa incidência de VMP nos pacientes neuropatas do presente estudo é similar aos dados descritos em outros países como Argentina (14\%), Reino Unido $(6 \%)^{16}$ e Brasil (10\%) $)^{5,17,18}$, porém, as incidências citadas da literatura não se relacionaram diretamente a pacientes neurológicos. Ademais, nas evidências disponíveis na literatura, a VMP foi definida como VM maior que 21 dias, entretanto, estes estudos utilizaram amostras heterogêneas em relação aos diagnósticos clínicos.

Muzaffar et al. 4 identificaram em uma coorte prospectiva de um ano, 49 pacientes (29\%) que permaneceram em VMP, com média de idade de 49,7 anos, $63 \%$ do sexo masculino, com mediana de tempo de VM de 39 dias e de internação na UTI de 39 dias; o desmame ventilatório obteve sucesso em $87 \%$. Esses achados são similares aos da presente pesquisa em alguns pontos, como a prevalência do sexo masculino e a faixa etária dos pacientes, porém, foram contrários em outros, como a incidência de VMP, o tempo de permanência em VM, o percentual de sucessos no desmame e o tempo de internação na unidade, que, no estudo atual, foram menores do que no estudo citado. Este fato pode ser explicado pela homogeneidade de pacientes e patologias incluídos no estudo presente, onde, a elegibilidade por pacientes neurológicos apenas, demonstrou características epidemiológicas similares.

Um estudo multicêntrico de coorte prospectiva ${ }^{5}$ identificou incidência de $10 \%$ de pacientes que necessitaram de VMP, definida como maior que 21 dias, com aumento significativo na mortalidade na UTI e maior tempo de

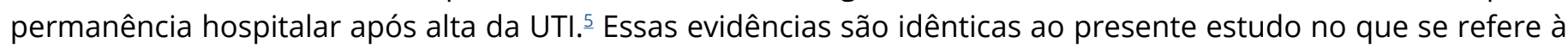
incidência de VMP, porém, foram contrárias no que tange ao período definido como VMP e o desfecho mortalidade, pois no nosso estudo a VMP foi definida como necessidade de VM maior ou igual a sete dias e não houve diferença na mortalidade na UTI entre os grupos com e sem VMP. A ausência de significância estatística nas taxas de mortalidade do presente estudo, em comparação ao estudo citado, pode ser explicada em partes, pelo tempo definido como VMP entre os dois estudos, ficando claro que quanto maior o tempo de permanência em suporte ventilatório invasivo, maiores as chances de piores desfechos.

Loss et al. $\underline{5}$ identificaram algumas complicações que se associaram a um risco maior de VMP, como fraqueza muscular, úlceras de pressão, sepse nosocomial bacteriana, candidemia, embolia pulmonar e delirium hiperativo, porém, estas não foram avaliadas na pesquisa atual. 
Um estudo recente ${ }^{19}$ avaliou a associação entre falência na extubação e desfechos funcionais em pacientes com doenças neurológicas agudas. Os pacientes tinham idade mediana de 58,5 anos, $60,5 \%$ do sexo masculino. Falha na extubação ocorreu em 12,8\% dos pacientes. A falha de extubação foi associada a maior tempo de permanência na UTI e no hospital e piores resultados funcionais em seis meses, conforme medido pelo escore de Rankin modificado. ${ }^{17}$

Piotto et al. $\frac{13}{}$ avaliaram os preditores para VMP, definida como maior que 48 horas, em pacientes submetidos à cirurgia de revascularização do miocárdio (CRM) e identificaram incidência de VMP em 3\% destes pacientes. Os autores identificaram idade, insuficiência renal crônica, doença pulmonar obstrutiva crônica, CRM associada a outros procedimentos e tempo de pinçamento como preditores independentes de VMP. Os achados desses autores contradizem os encontrados no presente estudo em relação à incidência de VMP, porém não são plausíveis de comparações pelas diferenças na definição de VMP entre os estudos e no tipo de pacientes cirúrgicos incluídos em cada estudo.

Segundo estudos prévios, entre $38-68 \%$ dos pacientes em VMP evoluem com sucesso no desmame ventilatório. ${ }^{20-22}$ Damuth et al. ${ }^{16}$ conduziram uma revisão sistemática com metanálise de 124 estudos de pacientes com insuficiência respiratória em VMP, definida como VM superior a 14 dias, dos quais, 50\% obtiveram desmame bem-sucedido. Essas evidências corroboram o presente estudo no qual $64 \%$ dos pacientes sob VMP concluíram o desmame ventilatório com êxito.

Os protocolos de desmame para pacientes sob VM convencional não se aplicam a pacientes sob VMP.른 Existem algumas evidências sobre estratégias de desmame em pacientes submetidos a VMP $\underline{422} 2 \underline{24}, 25$, porém, não há uma uniformidade de protocolos, bem como do tempo necessário para completar o desmame ventilatório, o que demonstra a dificuldade em prever o sucesso do desmame da VM. Tal fato demonstra a lacuna presente na literatura atual e necessidade de novos estudos sobre o tema.

Algumas limitações inerentes ao presente estudo se referem à ausência de acompanhamento em longo prazo dos pacientes para identificar desfechos clínicos importantes como mortalidade pós-alta hospitalar, a ausência da estratificação do uso de medicamentos como sedativos, drogas vasoativas e bloqueadores neuromusculares, pois, os mesmos interferem diretamente na evolução do desmame, assim como, o desenho metodológico retrospectivo do estudo, que permitiu falhas na inclusão de dados, devido sistemas incompletos e não incluiu análises estatísticas específicas para identificar preditores associados a VMP nos pacientes neurológicos. Além disso, outra limitação se refere à variedade de definições de VMP na literatura que dificulta a comparação entre os estudos.

Pode-se citar como implicações clínicas ou pontos fortes do presente estudo, o delineamento do perfil epidemiológico de pacientes com doenças neurológicas que evoluem para VMP, a fim de traçar estratégias de intervenção direcionadas para esse grupo de pacientes para acelerar o processo de desmame ventilatório e reduzir a incidência de morbidades e mortalidade.

\section{Conclusão}

A incidência de VMP foi de $10 \%$ nos pacientes com doenças neurológicas incluídos neste estudo. O grupo de pacientes sob VMP permaneceu mais tempo internado na UTI que o grupo sem suporte ventilatório prolongado, porém, não houve diferenças estatísticas no desfecho mortalidade e alta na UTI.

\section{Contribuições dos autores}

Ferreira LL, Sousa ACM e Sanchez LCA participaram da concepção, delineamento, busca e análise estatística dos dados da pesquisa, interpretação dos resultados, redação do artigo científico.

\section{Conflitos de interesses}

Nenhum conflito financeiro, legal ou político envolvendo terceiros (governo, empresas e fundações privadas, etc.) foi declarado para nenhum aspecto do trabalho submetido (incluindo, mas não se limitando a subvenções e financiamentos, participação em conselho consultivo, desenho de estudo, preparação de manuscrito, análise estatística, etc.). 


\section{Referências}

1. Santos CD, Nascimento ERPD, Hermida PMV, Silva TG, Galetto SGDS, Silva NJCD, et al. Boas práticas de enfermagem a pacientes em ventilação mecânica invasiva na emergência hospitalar. Esc Anna Nery. 2020;24(2):1-7. https://doi.org/10.1590/2177-9465EAN-2019-0300

2. Kutchak FM, Debesaitys AM, Rieder MDM, Meneguzzi C, Skueresky AS, Forgiarini-Junior LA, et al. PFE para tosse reflexa como preditor de extubação bem sucedida em pacientes neurológicos. J Bras Pneumol. 2015;41(4):358-64. https://doi. org/10.1590/S1806-37132015000004453

3. MacIntyre NR, Epstein SK, Carson S, Scheinhorm D, Christopher $\mathrm{K}$, Muldoon S, et al. Management of patients requiring prolonged mechanical ventilation: report of a NAMDRC consensus conference. Chest. 2005;128(6):3937-54. https://doi.org/10.1378/ chest.128.6.3937

4. Muzaffar SN, Gurjar M, Baronia AK, Azim A, Mishra P, Poddar B. Preditores, padrão de desmame e desfecho em longo prazo de pacientes com ventilação mecânica prolongada em unidade de terapia intensiva no norte da Índia. Rev Bras ter intensiva [Internet]. 2017;29(1):23-33. Disponível em: https://www.scielo. br/j/rbti/a/3JLV5hPw4mdRFyVDsRtz49S/?lang=pt

5. Loss SH, Oliveira RP, Maccari JG, Savi A, Boniatti MM, Hetzel MP, et al. A realidade dos pacientes que necessitam de ventilação mecânica prolongada: um estudo multicêntrico. Rev Bras Ter Intensiva. 2015;27(1):26-35. https://doi.org/10.5935/0103507X.20150006

6. Chelluri L, Im KA, Belle SH, Schulz R, Rotondi AJ, Donahoe MP, et al. Long-term mortality and quality of life after prolonged mechanical ventilation. Crit Care Med. 2004;32(1):61-9. https://doi. org/10.1097/01.CCM.0000098029.65347.F9

7. Sun Y, Li S, Wang S, Li C, Li G, Xu J, et al. Predictors of 1-year mortality in patients on prolonged mechanical ventilation after surgery in intensive care unit: a multicenter, retrospective cohort study. BMC Anesthesiol. 2020;20(1):44. https://doi.org/10.1186/ s12871-020-0942-0

8. MacIntyre NR, Epstein SK, Carson S, Scheinhorn D, Christopher $\mathrm{K}$, Muldoon S. Management of patients requiring prolonged mechanical ventilation: report of a NAMDRC consensus conference. Chest. 2005;128(6):3937-54. https://doi.org/10.1378/ chest.128.6.3937

9. Gracey DR, Hardy DC, Koenig GE. The chronic ventilatordependent unit: a lower-cost alternative to intensive care. Mayo Clin Proc. 2000;75(5):445-9. https://doi.org/10.4065/75.5.445

10. Bureau of Data Management and Strategy. 100\% MEDPAR inpatient hospital fiscal year 1998, 6/99 update. United States Health Care Finance Administration. Washington, DC: US Government Printing Office; 1999.
11. Gutiérrez EP, Díaz JSS, Hernandez RF, Rodriguez EAM, Peniche KG, Gutiérrez SPD, et al. Los predictores en el retiro de la ventilación mecánica resultan suficientes para el paciente neurocrítico? Med Int México. 2017;33(5):675-91. https://doi. org/10.24245/mim.v33i5.1561

12. Sousa ACM, Sanchez LCA, Ferreira LL. Desfechos clínicos de pacientes submetidos à ventilação mecânica invasiva em uma uti neurocirúrgica. ASSOBRAFIR Ciênc. 2021;12:e42286. https://doi. org/10.47066/2177-9333.AC.2020.0021

13. Piotto RF, Ferreira FB, Colósimo FC, Silva GS, Sousa AG, Braile DM. Fatores preditores independentes de ventilação mecânica prolongada em pacientes submetidos à cirurgia de revascularização miocárdica. Rev Bras Cir Cardiovasc [Internet]. 2012;27(4):520-8. Disponível em: https://www.scielo.br/j/rbccv/a/ xcJHcfcPJKQwncnWrqdBcWD/?format=pdf\&lang=en

14. Silva Júnior JM, Malbouisson LMS, Nuevo HL, Barbosa LGT, Maruabayeshi LY, Teixeira IC, et al. Aplicabilidade do escore fisiológico agudo simplificado (SAPS 3) em hospitais brasileiros. Rev Bras Anestesiol. 2010;60(1):20-31. https://doi.org/10.1590/ S0034-70942010000100003

15. Le Gall JR, Loirat P, Alperovitch A, Glaser P, Granthil C, Mathieu $D$, et al. A simplified acute physiology score for ICU patients. Crit Care Med. 1984;12(11):975-7. https://doi.org/10.1097/00003246198411000-00012

16. Damuth E, Mitchell JA, Bartock JL, Roberts BW, Trzeciak S. Long-term survival of critically ill patients treated with prolonged mechanical ventilation: a systemic review and meta-analysis. Lancet Respir Med. 2015;3(7):544-53. https://doi.org/10.1016/ $\underline{\mathrm{S} 2213-2600(15) 00150-2}$

17. Lone NI, Walsh TS. Prolonged mechanical ventilation in critically ill patients: epidemiology, outcomes and modelling the potential cost consequences of establishing a regional weaning unit. Crit Care. 2011;15(2):R102. https://doi.org/10.1186/cc10117

18. Estenssoro E, González F, Laffaire E, Canales H, Sáenz G, Reina $\mathrm{R}$, et al. Shock on admission day is the best predictor of prolonged mechanical ventilation in the ICU. Chest. 2005;127(2):598-603. https://doi.org/10.1378/chest.127.2.598

19. Rish MA, Kashyap R, Wilson G, Schenck L, Hocker S. Association of extubation failure and functional outcomes in patients with acute neurologic illness. Neurocrit Care. 2016;24(6):217-25. https://doi.org/10.1007/s12028-015-0156-3

20. Scheinhorn DJ, Hassenpflug MS, Votto J, Chao DC, Epstein SK, et al. Post-ICU mechanical ventilation at 23 long-term care hospitals: a multicenter outcomes study. Chest. 2007;131(1):8593. https://doi.org/10.1378/chest.06-1081

21. Scheinhorn DJ, Hassenpflug MS, Votto JJ, Chao DC, Epstein SK, Doig GS, et al. Ventilator-dependent survivors of catastrophic illness transferred to 23 long-term care hospitals for weaning from prolonged mechanical ventilation. Chest. 2007;131(1):76-84. https://doi.org/10.1378/chest.06-1079 
22. Bigatello LM, Stelfox HT, Berra L, Schmidt U, Gettings EM. Outcome of patients undergoing prolonged mechanical ventilation after critical illness. Crit Care Med. 2007;35(11):2491-7. https://doi.org/10.1097/01.CCM.0000287589.16724.B2

23. Huang CT, Yu CJ. Conventional weaning parameters do not predict extubation outcome in intubated subjects requiring prolonged mechanical ventilation. Respir Care. 2013;58(8):130714. https://doi.org/10.4187/respcare.01773
24. Jubran A, Grant BJ, Duffner LA, Collins EG, Lanuza DM, Hoffman LA, et al. Effect of pressure support vs unassisted breathing through a tracheostomy collar on weaning duration in patients requiring prolonged mechanical ventilation: a randomized trial. JAMA. 2013;309(7):671-7. https://doi. org/10.1001/jama.2013.159

25. Robertson TE, Sona C, Schallom L, Buckles M, Cracchiolo L, Schuerer D, et al. Improved extubation rates and earlier liberation from mechanical ventilation with implementation of a daily spontaneous-breathing trial protocol. J Am Coll Surg. 2008;206(3):489-95. https://doi.org/10.1016/j. jamcollsurg.2007.08.022 